\title{
Efeitos dos Choques Fiscais sobre o Mercado de Trabalho Brasileiro*
}

\author{
Diogo Baerlocher Carvalho ${ }^{\dagger}$, Marcelo Eduardo Alves da Silva ${ }^{\ddagger}$, Igor \\ Ézio Maciel Silva ${ }^{\S}$
}

\author{
Conteúdo: 1. Introdução; 2. Modelo Teórico; 3. Estimação do Modelo DSGE; 4. Estratégia \\ Empírica; 5. Resultados; 6. Análise de Robustez; 7. Considerações Finais; \\ A. Testes de Especificação do Modelo Empírico; B. Análise de Robustez. \\ Palavras-chave: Choques Fiscais, Mercado de Trabalho, Econometria Bayesiana. \\ Códigos JEL: C11, E24, E62.
}

Esse trabalho tem por objetivo entender quais os efeitos dos choques fiscais sobre o mercado de trabalho brasileiro. Em particular, utilizase uma abordagem SVAR para avaliar esses efeitos sobre o emprego e o salário real. Os choques fiscais são identificados a partir da imposição de restrições de sinais puras sobre a relação dinâmica entre os gastos do governo, o produto real e a oferta de trabalho. As restrições são obtidas a partir de funções de resposta a impulso Bayesianas derivadas de um modelo DSGE na tradição da Nova Síntese Neoclássica. As funções de resposta a impulso obtidas a partir da abordagem SVAR mostram que choques fiscais aumentam o salário real e o emprego.

This paper aims to understand what are the effects of fiscal shocks on the Brazilian labor market. In particular, we use a SVAR approach to analyze these effects on employment and real wages. Fiscal shocks are identified through the imposition of pure sign restrictions on the dynamic relationships between government spending, real product and labor supply. The restrictions are obtained from an estimated DSGE model in the New Neoclassical Synthesis tradition. Our results from estimated impulse response functions show that following a fiscal shock, both employment and real wages increase.

\footnotetext{
* Os autores agradecem os valiosos comentários e sugestões de um parecerista anônimo e do editor associado Alexandre Cunha. Os eventuais erros remanescentes são de nossa responsabilidade. Marcelo agradece o apoio do CNPq.

${ }^{\dagger}$ Aluno do PhD em Economia da Universidade de Illinois at Urbana-Champaign.E-mail: dcarval2@illinois . edu

$¥$ Professor do Departamento de Economia da Universidade Federal de Pernambuco e do PIMES/UFPE.

E-mail: marcelo.easilva@ufpe.br

${ }^{\S}$ Doutorando em Economia do Programa de Pós-Graduação em Economia da UFPE. E-mail: igor.msilva@ufpe.br
} 


\section{INTRODUÇÃO}

A recente crise financeira internacional tem reacendido o debate sobre a importância das políticas macroeconômicas na estabilização da economia entre economistas e formuladores de políticas. Do ponto de vista da política monetária, o consenso em torno da utilização de uma política convencional como instrumento principal na ação de estabilização tem perdido força à medida que o atual cenário, onde as taxas de juros internacionais já se encontram em níveis suficientemente baixos, tem imposto limites à sua eficácia. Diante disto, a discussão tem sido em torno da implementação e da eficácia de medidas alternativas ou não-convencionais (e.g. quantitative easing), que em conjunto com medidas mais tradicionais, poderiam ser mais eficazes na tentativa de estabilização do produto e do emprego. Note, contudo, que o consenso em torno da direção de uma política monetária expansionista permanece, com aumentos no emprego e no produto consistindo nos resultados consensuais.

Por outro lado, mesmo antes da recente crise financeira internacional ter atingindo com força as economias dos países e as certezas em torno dos efeitos da política monetária, uma outra importante discussão existia na literatura em torno do papel da política fiscal. Se por um lado, existia (e talvez ainda exista) um certo consenso em torno dos efeitos da política monetária, o mesmo não pode ser dito em relação à política fiscal. Como ressaltado por Perotti (2007), os economistas discordam até mesmo dos efeitos básicos da política fiscal sobre a economia e por esta razão diversos trabalhos surgiram com o objetivo de entender (e mensurar) tais efeitos (e.g. Blanchard e Perotti (2002); Fatás e Mihov (2001); Mountford e Uhlig (2009)). A discordância se dá tanto do ponto de vista teórico quanto empírico.

Do ponto de vista teórico, os modelos dos Ciclos Reais de Negócios (RBC) e os Novos Keynesianos (NK) divergem quanto aos resultados de expansões nos gastos do governo sobre algumas variáveis macroeconômicas, notadamente sobre o consumo e o salário real. Dentro do arcabouço novo clássico, aumentos inesperados nos gastos do governo têm como resultado um efeito riqueza negativo causado pela perspectiva de impostos mais altos no futuro. Dessa forma, ocorre um aumento da oferta de trabalho e, consequentemente, uma redução no salário real. Por fim, o consumo privado diminui sendo praticamente substituído pelo consumo público. Em modelos na tradição Novo Keynesiana, a expansão dos gastos públicos causa igualmente uma redução no consumo privado e uma expansão na oferta de trabalho, porém também desloca a demanda por trabalho, resultando em uma elevação do salário real. Portanto, claramente, modelos nas duas tradições (RBC e NK) preconizam impactos diferenciados sobre o salário real. E estes resultados são diferentes basicamente em função do grau de rigidez que é assumido em cada modelo. Enquanto nos modelos RBC preços são flexíveis, nos modelos na tradição NK os preços apresentam algum tipo de rigidez.

Por outro lado, a evidência empírica não parece ser clara quanto aos efeitos de choques fiscais sobre a economia. Um elemento importante que explica os diferentes resultados consiste na forma como choques fiscais são identificados nos modelos empíricos, particularmente, nos modelos Estruturais AutoRegressivos Vetoriais - SVAR. Nesta questão, diferentes metodologias têm sido utilizadas na tentativa de entender os efeitos de choques fiscais. Tradicionalmente, o primeiro passo consistiu em utilizar uma abordagem narrativa (análise de eventos) na tentativa de identificar os choques fiscais. A ideia básica era a de que estes choques consistiam em alterações anormais no caminho natural das variáveis endógenas. Por exemplo, Ramey e Shapiro (1998) e Burnside et alii (2004) utilizam este método para analisar como a expansão nos gastos militares realizados pelo governo americano afetaram o produto, consumo e emprego, entre outras variáveis. Os impactos de alterações anormais é capturado a partir da inclusão de variáveis dummies no período de ocorrência desses eventos. Os resultados mostram quedas no consumo e no salário real seguindo um choque fiscal, o que corrobora com as implicações teóricas de modelos na tradição RBC.

Diferentemente do preconizado pelos trabalhos que utilizam a abordagem narrativa, aqueles que implementam uma abordagem de identificação de choques fiscais a partir da utilização de informações institucionais, impondo assim restrições de curto (ou longo) prazo na maneira como as variáveis macroeconômicas respondem a estes choques, obtêm resultados que dão suporte às implicações de modelos 
na tradição NK. Por exemplo, Blanchard e Perotti (2002) e Fatás e Mihov (2001) mostram que seguindo um choque fiscal, ambos consumo e salário real aumentam. Portanto, neste contexto, fica evidente a falta de consenso em torno dos impactos de choques fiscais sobre a economia.

Mais recentemente tem sido proposta uma identificação alternativa, que consiste em impor um conjunto mínimo de restrições sobre os sinais das respostas de algumas variáveis no modelo SVAR e com isto encontrar as respostas das variáveis de interesse a choques fiscais. Esta metodologia alternativa foi proposta por Uhlig (2005) no contexto de choques monetários. Um problema inicial neste tipo de identificação era que em muitos casos as restrições de sinal (direção das respostas) eram feitas de maneira informal, com base na crença do pesquisador sobre como deveriam responder algumas variáveis a choques fiscais (ou qualquer outro choque). A partir disto, um melhoramento nesta abordagem consistiu em derivar os sinais a partir da formulação de um modelo de Equilíbrio Geral Dinâmico e Estocástico (DSGE) e com isto ser capaz de identificar os impactos de choques fiscais sobre as variáveis de interesse, que são deixadas livres durante o processo de estimação. Em seus resultados a partir da utilização da referida metodologia, Mountford e Uhlig (2009) encontram que choques fiscais estimulam fracamente o produto, enquanto a resposta do consumo é muito fraca, assim como encontrado por Burnside et alii (2004). Estes resultados parecem não oferecer suporte nem às implicações de modelos na tradição RBC nem àquelas dos modelos na tradição NK.

Para o caso brasileiro, Peres e Ellery Jr (2009) utilizam informações institucionais com o objetivo de identificar os choques fiscais. Estes autores encontram resultados consistentes com o modelo NK, em particular, o produto apresenta uma resposta positiva ao choque fiscal. Mendonça et alii (2010) utilizaram a estratégia de identificação agnóstica para investigar os efeitos dos choques fiscais na economia brasileira. As respostas a aumentos inesperados dos gastos do governo foram positivas para o consumo e taxa de juros, enquanto o PIB apresentou declínio.

Apenas mais recentemente é que a literatura internacional tem focado interesse em conhecer os impactos de choques fiscais sobre variáveis do mercado de trabalho, particularmente utilizando a metodologia SVAR com restrições de sinais. O primeiro trabalho com foco particular neste aspecto é o de Pappa (2009), que investiga como o nível de emprego e salário real reagem a choques fiscais com dados norte-americanos. O método é baseado em Canova (2002), em que são aplicadas restrições de sinal sobre as respostas tomando por base um Modelo de Equilíbrio Geral Dinâmico e Estocástico (DSGE). A vantagem desta metodologia é que o modelo DSGE proporciona restrições exatas para o comportamento das variáveis, permitindo assim uma melhor identificação do que seriam os verdadeiros choques fiscais nos dados.

O objetivo principal desse trabalho é lançar evidências empíricas sobre o impacto de choques fiscais sobre as variáveis de mercado de trabalho, em particular sobre o salário real, utilizando dados brasileiros. Este objetivo principal é alcançado através da estimação de um modelo Estrutural Auto-Regressivo Vetorial e da posterior análise das funções de resposta à impulso. A identificação do modelo SVAR será feita a partir do método proposto por Canova (2002) e aplicada por Dedola e Neri (2007) e Pappa (2009) que consiste na utilização de um modelo DSGE na derivação de restrições que possibilitem a identificação de choques fiscais.

O modelo DSGE utilizado neste artigo é o mesmo de Smets e Wouters (2003), o qual segue a tradição da Nova Síntese Neoclássica e é geral o suficiente para incluir uma situação com preços flexíveis (RBC) e preços rígidos (Novo Keynesiano) como casos especiais, não impondo, portanto, nenhuma restrição a priori do que se esperam ser os impactos dos choques fiscais sobre as variáveis de interesse. Vale notar que a literatura teórica analisando os impactos de choques fiscais na economia brasileira ainda é incipiente, com alguns poucos trabalhos sendo identificados na literatura, por exemplo, Silva e Portugal (2010), Castro et alii (2011) e Gadelha e Divino (2012).

O modelo DSGE é então estimado utilizando técnicas de inferência Bayesiana (Cadeias de Markov Monte Carlo - MCMC) com base em dados brasileiros. Uma vez estimado o modelo, o passo seguinte consistiu em observar as implicações das duas versões do modelo (preços flexíveis e preços rígidos) no intuito de identificar variáveis cujos comportamentos sejam qualitativamente semelhantes sob ambas 
as hipóteses e com isto impor restrições mínimas sobre estas variáveis no modelo SVAR, ao mesmo tempo em que se é agnóstico na maneira como as variáveis de interesse respondem.

Os resultados do modelo teórico indicaram que, sob ambas as hipóteses de rigidez de preços, choques fiscais afetam de forma positiva o produto e o emprego na economia. No entanto, o efeito dos choques fiscais sobre o salário real foram contraditórios. Enquanto no modelo com preços rígidos o salário real sofre impacto positivo, no modelo com preços flexíveis, o salário real cai frente a um choque fiscal. Portanto, embora possamos impor restrições exatas sobre o comportamento do PIB e da oferta de trabalho com base no modelo DSGE, somos completamente agnósticos na maneira como o salário real responde a choques fiscais.

Dessa forma, o choque fiscal é definido como uma vetor-impulso cujas respostas sobre os gastos do governo, produto e a oferta de trabalho sejam não-negativas e a partir disto construímos as funções de resposta a impulso para as variáveis do mercado de trabalho. Os resultados mostraram que em resposta a um choque fiscal, o salário real aumenta, o que indica que choques fiscais deslocam a demanda por trabalho e possuem, portanto, caráter Novo Keynesiano no Brasil.

Além desta introdução, o artigo apresenta seis outras seções. Na seção seguinte é apresentado o modelo teórico em que se baseiam as restrições de sinal. Em seguida, a estratégia de estimação dos parâmetros estruturais do modelo DSGE é discutida, assim como os principais resultados da estimação. Esta seção discute ainda as implicações derivadas a partir do modelo DSGE e que são empregadas na estratégia empírica. A Seção 4 apresenta e discute a estratégia empírica empregada neste artigo. Em particular, apresenta as bases de dados utilizadas, a metodologia SVAR e o processo de identificação dos choques estruturais utilizando o método de restrição de sinais. A Seção 5 mostra e discute os principais resultados da estratégia empírica. Em seguida, são apresentados alguns resultados da análise de robustez. Por fim, são tecidas as considerações finais deste trabalho.

\section{MODELO TEÓRICO}

O modelo de Equilíbrio Geral Dinâmico e Estocástico utilizado é o mesmo de Smets e Wouters (2003), que consiste em um modelo dentro da Nova Síntese Neoclássica tendo como casos particulares tanto uma economia com preços flexíveis (RBC) quanto uma com preços e salários rígidos (NK). As famílias nesta economia maximizam o valor presente descontado da utilidade esperada escolhendo os níveis de consumo, trabalho, títulos, investimento, utilização de capital, e estoque de capital. Trabalho nesta economia é um bem diferenciado, o que garante às famílias um certo poder de monopólio na determinação dos salários. Existem dois tipos de firmas, uma produzindo um bem final e outra produzindo bens intermediários. A autoridade monetária estabelece a taxa de juros nominal de maneira a estabilizar o produto e a inflação, e a autoridade fiscal determina o nível de gastos em bens e serviços do governo. A seguir descrevemos o comportamento de cada um dos agentes da economia.

\subsection{Famílias}

A economia é composta por um contínuo de famílias indexadas pela letra $i$. Cada família $i$ resolve um problema de otimização intertemporal escolhendo os níveis de consumo, $C_{t}^{i}$, trabalho, $\ell_{t}^{i}$, investimento, $I_{t}^{i}$, títulos, $B_{t}^{i}$, utilização do capital, $z_{t}^{i}$, estoque de capital, $K_{t}^{i}$ e salários nominais, $w_{t}^{i}$, que maximizam o valor presente descontado da utilidade esperada sujeita a restrição orçamentária intertemporal e algumas outras restrições que serão apresentadas a seguir em mais detalhes.

A função objetivo de cada família $i$ é dada por

$$
\mathbb{E}_{0} \sum_{t=0}^{\infty} \beta^{t} U_{t}^{i},
$$


em que

$$
U_{t}^{i}=\varepsilon_{t}^{b}\left[\frac{1}{1-\sigma_{c}}\left(C_{t}^{i}-\lambda C_{t-1}^{i}\right)^{1-\sigma_{c}}-\frac{\varepsilon_{t}^{L}}{1+\sigma_{\ell}}\left(\ell_{t}^{i}\right)^{1+\sigma_{\ell}}\right]
$$

O parâmetro $\lambda$ governa o grau de formação de hábito no consumo, $\sigma_{c}$ é o inverso da elasticidade de substituição intertemporal e $\sigma_{\ell}$ é o inverso da elasticidade do trabalho em relação ao salário real. A função de utilidade apresenta um choque de preferência, $\varepsilon_{t}^{b}$, e um choque na oferta de trabalho, $\varepsilon_{t}^{L}$. Ambos seguem um processo autoregressivo de primeira ordem com termos de erros sendo dados, respectivamente, por $\eta_{t}^{b} \sim N\left(0, \sigma_{b}\right)$ e $\eta_{t}^{E L} \sim N\left(0, \sigma_{E L}\right)$.

A restrição orçamentária intertemporal enfrentada por cada família $i$ é dada por:

$$
C_{t}^{i}+I_{t}^{i}+b_{t} \frac{B_{t}^{i}}{P_{t}}+\Psi\left(z_{t}^{i}\right) K_{t-1}^{i} \leq \frac{B_{t-1}^{i}}{P_{t}}+\frac{w_{t}^{i}}{P_{t}} \ell_{t}^{i}+A_{t}^{i}+\frac{r_{t}^{k}}{P_{t}} z_{t}^{i} K_{t-1}^{i}+\frac{d_{t}^{i}}{P_{t}}+\frac{T_{t}}{P_{t}},
$$

em que $w_{t}$ é o salário recebido pelas famílias, $r_{t}^{k}$ é a taxa de aluguel do capital, $d_{t}$ representa os dividendos recebidos das firmas intermediárias, $B_{t}$ são títulos nominais que possuem maturidade de um período com preço $b_{t}$, onde $b_{t}=R_{t}^{-1}$ e $R_{t}$ é a taxa de juros nominal, $P_{t}$ é o nível de preços agregado e $A_{t}$ é a renda líquida recebida pela família $i$ por possuir títulos estado-dependentes. ${ }^{1}$ O lado esquerdo da restrição orçamentária revela os usos dos recursos, enquanto o lado direito as fontes de recursos das famílias. Note ainda que a renda do capital depende não apenas do estoque de capital, $K_{t-1}^{i}$, mas também do grau de utilização do capital, $z_{t}^{i}$. As duas variáveis são escolhidas pelas famílias no processo de otimização intertemporal.

As famílias são as proprietárias do estoque de capital, que é alugado às firmas de bens intermediários à taxa $r_{t}^{k}$. As famílias podem alterar os "serviços"do capital de duas formas: ou através de novo investimento ou alterando a taxa de utilização do capital já existente, $z_{t}$. Portanto, as famílias escolhem os níveis de investimento, estoque de capital e taxa de utilização do capital de maneira a maximizar a função objetivo (1) sujeita a restrição orçamentária (2) e à lei de movimento do estoque de capital:

$$
K_{t}=(1-\delta) K_{t-1}+\left[1-S\left(\frac{I_{t}}{I_{t-1}}\right)\right] I_{t},
$$

em que $\delta$ é taxa de depreciação do capital físico e $S(\cdot)$ é uma função que captura o custo de ajustamento do investimento, onde $S(\cdot)=0$ e $S^{\prime}(\cdot)=0$ no estado estacionário e $S^{\prime \prime}(\cdot)>0$.

Ademais cada família $i$ é proprietária de uma unidade de trabalho levemente diferenciado, o qual é ofertado para as firmas que produzem bens intermediários. A diferenciação do trabalho confere algum poder de monopólio sobre os salários e possibilita a introdução de salário nominais rígidos à la Calvo (1983). Isto significa dizer que em cada momento no tempo, apenas uma fração $1-\xi_{w}$ das famílias podem escolher salários nominais de maneira ótima. As famílias que não têm essa possibilidade indexam seus salários à inflação do período anterior de forma que

$$
w_{t}^{i}=\left(\frac{P_{t-1}}{P_{t-2}}\right)^{\gamma_{w}} w_{t-1}^{i}
$$

em que $\gamma_{w}$ é o grau de indexação permitido.

Portanto, o problema de otimização intertemporal das famílias também inclui a escolha de salários nominais ótimos. Esta escolha é feita de maneira a maximizar a função objetivo (1) sujeita a restrição orçamentária (2) e à demanda por trabalho, que é dada por

$$
\ell_{t}^{i}=\left(\frac{w_{t}^{i}}{W_{t}}\right)^{-\left(1+\lambda_{t}^{w}\right) / \lambda_{t}^{w}} L_{t}
$$

\footnotetext{
${ }^{1}$ Como destacado por Smets e Wouters (2003), estes títulos são uma forma de proteger as famílias contra flutuações na renda do trabalho fruto de choques idiossincráticos.
} 
em que $L_{t}$ é a demanda agregada por trabalho e $W_{t}$ é o salário nominal agregado. Estas duas variáveis são agregadas entre as diferentes famílias da seguinte maneira:

$$
L_{t}=\left[\int_{0}^{1}\left(\ell_{t}^{i}\right)^{1 /\left(1+\lambda_{t}^{w}\right)} d i\right]^{1+\lambda_{t}^{w}}
$$

e

$$
W_{t}=\left[\int_{0}^{1}\left(w_{t}^{i}\right)^{-1 / \lambda_{t}^{w}} d i\right]^{-\lambda_{t}^{w}},
$$

em que $\lambda_{t}^{w}$ é o mark-up do salário. O mark-up segue um processo tal que $\lambda_{t}^{w}=\lambda^{w}+\eta_{t}^{w}$.

Do problema de escolha dos salários ótimos resulta a lei de movimento do salário que é dada por:

$$
\left(W_{t}\right)^{-1 / \lambda_{t}^{w}}=\xi_{w}\left(W_{t-1}\left(\frac{P_{t-1}}{P_{t-2}}\right)^{\gamma_{w}}\right)^{-1 / \lambda_{t}^{w}}+\left(1-\xi_{w}\right)\left(\tilde{w}_{t}\right)^{-1 / \lambda_{t}^{p}},
$$

em que $\tilde{w}_{t}$ é salário re-otimizado.

\subsection{Firmas}

Existem dois tipos de firmas nesta economia: firmas que produzem um bem final agregado, o qual é utilizado para consumo e investimento, e firmas que produzem bens intermediários, os quais são utilizados na produção do bem final. As firmas de bem final atuam em um mercado perfeitamente competitivo, enquanto aquelas que atuam no setor de bens intermediários atuam sob competição monopolística.

\subsubsection{Produção do Bem Final}

O bem final $Y_{t}$ é produzido a partir da agregação de um contínuo de bens intermediários $y_{t}^{j}$. A tecnologia de produção do bem final é tal que

$$
Y_{t}=\left[\int_{0}^{1}\left(y_{t}^{j}\right)^{1 /\left(1+\lambda^{p}\right)} d j\right]^{1+\lambda^{p}}
$$

em que $y_{t}^{j}$ é a quantidade do bem intermediário $j$ usado na produção de bem final e $\lambda^{p}$ é o mark-up do mercado de bens.

Do processo de minimização de custos por parte das firmas finais, é possível obter a seguinte escolha ótima de bens intermediários:

$$
y_{t}^{i}=\left(\frac{p_{t}^{j}}{P_{t}}\right)^{-\left(1+\lambda^{p}\right) / \lambda^{p}} Y_{t}
$$

em que $p_{t}^{j}$ é o preço do bem intermediário $j$ e $P_{t}$ é tal que

$$
P_{t}=\left[\int_{0}^{1}\left(p_{t}^{j}\right)^{-1 / \lambda^{p}} d j\right]^{-\lambda^{p}}
$$

\subsubsection{Firmas de Bens Intermediários}

Nesta economia existem um contínuo de firmas intermediárias cada uma produzindo um bem intermediário levemente diferenciado. Cada bem diferenciado $j$ é produzindo de acordo com a seguinte tecnologia:

$$
y_{t}^{j}=\varepsilon_{t}^{a}\left(K_{t}^{s j}\right)^{\alpha}\left(L_{t}^{j}\right)^{1-\alpha}-\Phi
$$


em que $K_{t}^{s}=z_{t} K_{t-1}$ é o capital efetivo usado pelas firmas, $L_{t}^{j}$ é um índice de utilização de trabalho diferenciado por parte das firmas dado por (3), $\Phi$ é um custo fixo e $\varepsilon_{t}^{a}=\rho_{a} \varepsilon_{t-1}^{a}+\eta_{t}^{a}$ é um choque de produtividade que segue um processo autoregressivo de primeira ordem com termo de erro $\eta_{t}^{a} \sim$ $N\left(0, \sigma_{a}\right)$.

A combinação das escolhas ótimas das firmas intermediárias de trabalho, $L_{t}^{j}$, e capital físico, $K_{t}^{s}$, produz:

$$
\frac{W_{t} L_{t}^{j}}{r_{t}^{k} K_{t}^{s}}=\frac{1-\alpha}{\alpha}
$$

Esta condição implica que as escolhas ótimas de trabalho e capital serão idênticas dentre as firmas. 0 custo marginal também será idêntico dentre as firmas e pode ser representado como

$$
M C_{t}=\left[\alpha^{-\alpha}(1-\alpha)^{-(1-\alpha)}\right] W_{t}^{1-\alpha}\left(r_{t}^{k}\right)^{\alpha}\left(\varepsilon_{t}^{a}\right)^{-1}
$$

É possível então escrever o lucro nominal das firmas intermediárias em $t$ como:

$$
\pi_{t}^{j}=\left(p_{t}^{j}-M C_{t}\right) Y_{t}\left(\frac{p_{t}^{j}}{P_{t}}\right)^{-\left(1+\lambda_{t}^{p}\right) / \lambda_{t}^{p}}-M C_{t} \Phi
$$

em que $p_{t}^{j}$ é o preço do bem intermediário.

Como no caso das famílias, as firmas não podem reajustar o preço do bem intermediário a menos que recebam um sinal para tanto. E este sinal é recebido com probabilidade $1-\xi_{p}$. As firmas que não podem re-otimizar seus preços em um determinado momento, determinam preço a partir da inflação do período anterior, num mecanismo de indexação. Portanto, em cada momento $t$ um subconjunto de firmas determina preços de maneira ótima, enquanto o subconjunto restante determina preços indexandoos à inflação passada.

Quando permitidas reajustar preços, as firmas intermediárias o fazem tal que a condição

$$
\mathbb{E}_{t} \sum_{h=0}^{\infty} \beta^{h} \xi_{p}^{h} \lambda_{t+h} y_{t+h}^{j}\left[\frac{\tilde{p}_{t}^{j}}{P_{t}}\left(\frac{\left(P_{t-1+h} / P_{t-1}\right)^{\gamma^{p}}}{P_{t+h} / P_{t}}\right)-\left(1+\lambda_{t+h}^{p}\right) m c_{t+h}\right]=0
$$

é satisfeita. A lei de movimento do índice de preços é dada por:

$$
\left(P_{t}\right)^{-1 / \lambda_{t}^{p}}=\xi_{p}\left(P_{t-1}\left(\frac{P_{t-1}}{P_{t-2}}\right)^{\gamma_{p}}\right)^{-1 / \lambda_{t}^{p}}+\left(1-\xi_{p}\right)\left(\tilde{p}_{t}^{j}\right)^{-1 / \lambda_{t}^{p}}
$$

em que $\gamma_{p}$ é o nível de indexação.

\subsection{Governo}

A autoridade fiscal compra o bem final, $G_{t}$, emite títulos, $B_{t}$, e cobra impostos do tipo lump sum, $T_{t}$ em cada período. A restrição orçamentária do governo é dada por:

$$
P_{t} G_{t}+B_{t-1}=P_{t} T_{t}+\frac{B_{t}}{R_{t}}
$$

Diferentemente de Smets e Wouters (2003) é assumido que $G_{t}$ é tal que:

$$
G_{t}=\rho_{g} G_{t-1}+\rho_{y}\left(Y_{t}-Y_{t-1}\right)+\eta_{t}^{g}
$$


em que $\eta_{t}^{g} \sim N\left(0, \sigma_{g}\right)$ representa o termo de erro, e o termo $\rho_{y}$ governa a resposta dos gastos fiscais ao ciclo econômico. Este termo foi introduzido como forma de capturar, pelo menos em forma reduzida, a discussão em torno da contra-ciclicalidade ou não dos gastos fiscais em economias emergentes como a brasileira (ver por exemplo Frankel et alii (2011)). ${ }^{2}$

A autoridade monetária segue uma regra de taxa de juros, que responde gradualmente a desvios da inflação da meta estabelecida e a desvios do hiato do produto. Igualmente, é permitido que a autoridade monetária leve em conta mudanças recentes na taxa de inflação e no hiato do produto na determinação da taxa de juros nominal. Esta regra (na forma log-linear) pode ser descrita da seguinte maneira:

$$
\begin{aligned}
\hat{R}_{t}=\rho_{R} \hat{R}_{t-1}+\left(1-\rho_{R}\right)\left[\bar{\pi}_{t}+r_{\pi}\left(\hat{\pi}_{t-1}-\bar{\pi}_{t}\right)+r_{y}\right. & \left.\left(\hat{Y}_{t}-\hat{Y}_{t}^{*}\right)\right]+r_{\Delta \pi}\left(\hat{\pi}_{t}-\hat{\pi}_{t-1}\right)+ \\
& +r_{\Delta y}\left[\hat{Y}_{t}-\hat{Y}_{t}^{*}-\left(\hat{Y}_{t-1}-\hat{Y}_{t-1}^{*}\right)\right]+\eta_{t}^{R}
\end{aligned}
$$

o parâmetro $\rho_{R}$ representa o nível de suavização da taxa de juros, $r_{\pi}$ governa a resposta da autoridade monetária a desvios da inflação da meta, $\bar{\pi}, r_{y}$ governa a resposta ao hiato do produto, $r_{\Delta \pi}$ determina a resposta a mudanças na taxa de inflação, $r_{\Delta y}$ governa a resposta a mudanças no hiato do produto e $\eta_{t}^{R} \sim N\left(0, \sigma_{r}\right)$ representa o termo de erro da regra de Taylor.

\subsection{Equilíbrio de Mercado}

O mercado de bens finais está em equilíbrio quando oferta é igual a demanda tal que

$$
Y_{t}=C_{t}+G_{t}+I_{t}+\Psi\left(z_{t}\right) K_{t-1} .
$$

Além disso, é necessário que a demanda por capital por parte das firmas de bens intermediários se iguale a oferta por parte das famílias, da mesma forma que a oferta de trabalho deve igualar a demanda, equilibrando assim o mercado de trabalho.

\section{ESTIMAÇÃO DO MODELO DSGE}

A solução do modelo DSGE produz uma série de condições de primeira ordem não-lineares. De forma a encontrar a solução do modelo, nós calculamos uma aproximação de Taylor de primeira ordem das condições de equilíbrio ao redor do estado-estacionário não estocástico. A partir disto, foi utilizado o método proposto por Sims (2002) para encontrar a solução do modelo. A solução do modelo DSGE linearizado pode ser escrito da seguinte maneira:

$$
X_{t+1}=A(\theta) X_{t}+B(\theta) \omega_{t+1}
$$

onde $\omega_{t+1}$ descreve as inovações exógenas e $\omega_{t+1} \sim N(0, Q)$, and $X_{t}$ representa o vetor de variáveis do modelo. As matrizes $A(\theta)$ and $B(\theta)$ coleciona os parâmetros estruturais, onde $\theta=\left(\theta_{C}, \theta_{E}\right) \in \Theta$. O subconjunto $\theta_{C}$ descreve os parâmetros que são fixos e $\theta_{E}$ aqueles que são livres no processo de estimação.

Para estimação do modelo DSGE foram utilizadas 6 variáveis com periodicidade trimestral: PIB real, consumo das famílias, horas trabalhadas, gastos do governo, taxa de juros nominal e inflação. Dada a solução do modelo em forma de estado de espaço e o vetor de variáveis observáveis, é possível construir a função de verossimilhança $£\left(\Psi_{t} \mid \theta\right)$ com a ajuda do Filtro de Kalman. Distribuições de probabilidade a priori $p(\theta)$ são estabelecidas com base em crenças sobre a distribuição dos parâmetros.

\footnotetext{
${ }^{2}$ No processo de estimação do modelo DSGE, este parâmetro possui distribuição a priori uniforme, com média zero. Com isso deixamos os dados decidirem qual o valor do parâmetro mais apropriado.
} 
A partir destas distribuições a priori e da função de verossimilhança, o Teorema de Bayes permite a derivação da distribuição conjunta de probabilidade posterior da seguinte maneira:

$$
P\left(\theta \mid \Psi_{t}\right)=\frac{£\left(\Psi_{t} \mid \theta\right) p(\theta)}{\int_{\theta} £\left(\Psi_{t} \mid \theta\right) p(\theta) d(\theta)}
$$

onde $\int_{\theta} £\left(\Psi_{t} \mid \theta\right) p(\theta) d(\theta)$ descreve a densidade marginal dos dados.

O objetivo da estimação é obter a distribuição conjunta de probabilidade posterior dos parâmetros estruturais. Isto é realizado com base no algoritmo Metropolis-Hastings, o qual é um método de Cadeia de Markov Monte Carlo (MCMC) que permite a obtenção de sequências de qualquer distribuição de probabilidade e, em particular, daquelas que são não padronizadas.

A ideia por trás da MCMC é obter a kernel posterior que tem a distribuição posterior como sua distribuição invariante. Primeiro note que a densidade marginal dos dados quando integrada resulta numa constante e, portanto, toda a informação a cerca de $\theta$ a partir dos dados pode ser obtida através da Kernel posterior

$$
P\left(\theta \mid \Psi_{t}\right) \propto £\left(\Psi_{t} \mid \theta\right) p(\theta)
$$

$\mathrm{O}$ algoritmo Metropolis-Hastings gera retiradas da kernel posterior

$$
\log £\left(\Psi_{t} \mid \theta\right)+\log p(\theta)
$$

com o objetivo de derivar uma aproximação Normal da distribuição conjunta de probabilidade posterior ao redor da moda posterior. Para este fim foram geradas duas sequências independentes com cada uma sendo composta de 1.000 .000 retiradas usando o algoritmo de Metropolis-Hastings. A aceitação média ao longo das duas cadeias ficou em torno de $25 \%$, e convergência foi avaliada utilizando os métodos propostos por Brooks e Gelman (1998). As primeiras 500.000 retiradas foram descartadas para assegurar independência das condições iniciais. As estatísticas de interesse foram então calculadas com base na distribuição conjunta de probabilidade posterior ergódica dos parâmetros estruturais.

\subsection{Calibração e Distribuições a Priori}

Alguns parâmetros foram mantidos fixos durante o processo de estimação, enquanto outros foram estimados. A escolha dos valores para os parâmetros, tanto aqueles que permaneceram fixos quanto aqueles que foram estimados, foi baseada em três alternativas. A primeira, sempre que possível, foram utilizados valores para os parâmetros que apresentavam valores correspondentes nos dados brasileiros. Este foi o caso, por exemplo, das proporções dos gastos em consumo das famílias em relação ao PIB, e das proporções do investimento e dos gastos do governo no PIB.

A segunda alternativa foi a utilização de valores comumente utilizados na literatura correlata. Este foi o caso de parâmetros como o fator de desconto das famílias $\beta$, da proporção de capital físico na função de produção, $\alpha$, da taxa de depreciação, $\delta$. Por fim, para os parâmetros que foram estimados, na maior parte dos casos utilizamos distribuições de probabilidade a priori semelhantes às utilizadas por Smets e Wouters (2003), Smets e Wouters (2007), e Silva e Portugal (2010). A Tabela 1 apresenta uma descrição dos parâmetros estruturais do modelo DSGE.

\subsection{Resultados da Estimação}

Esta subseção apresenta os resultados da estimação do modelo DSGE. As Tabelas 2 e 3 apresentam os valores médios, os desvios padrão e os valores correspondentes ao intervalo de confiança dos parâmetros estimados utilizando a técnica de inferência Bayesiana.

A Figura 1 apresenta as respostas das variáveis de interesse a um choque nos gastos governamentais sob a hipótese de preços flexíveis, enquanto a Figura 2 apresenta as respostas das variáveis de interesse a um choque nos gastos governamentais sob a hipótese de preços rígidos. 
Tabela 1: Descrição dos Parâmetros

\begin{tabular}{|c|c|c|}
\hline Símbolo & Descrição & Valor \\
\hline$\beta$ & Fator de desconto & 0.99 \\
\hline$\sigma_{l}$ & Inversa da elasticidade do esforço do trabalho & 2.0 \\
\hline$\sigma_{c}$ & Coeficiente de aversão ao risco das famílias & 1.0 \\
\hline$\lambda$ & Parâmetro de persistência do hábito no consumo & 0.7 \\
\hline$\delta$ & Taxa de depreciação do capital & 0.025 \\
\hline$\alpha$ & Proporção de capital na função de produção & 0.30 \\
\hline $\bar{r}_{k}$ & Taxa de juros real no estado estacionário & $1 / \beta-1+\delta$ \\
\hline $\bar{c}$ & Proporção do consumo das famílias no PIB & 0.61 \\
\hline $\bar{i}$ & Proporção do investimento no PIB & 0.18 \\
\hline $\bar{g}$ & Proporção dos gastos do governo no PIB & 0.20 \\
\hline$\Phi$ & Custos fixos na produção & 1.408 \\
\hline$\phi_{i}$ & Inversa do custo de ajustamento do investimento & $1 / 6.771$ \\
\hline$\gamma_{p}$ & Grau de indexação dos preços & 0.75 \\
\hline$\gamma_{w}$ & Grau de indexação dos salários & 0.75 \\
\hline$\lambda^{w}$ & Mark-up na determinação do salário & 0.5 \\
\hline$\xi_{p}$ & Parâmetro de Calvo nos preços & 0.75 \\
\hline$\xi_{w}$ & Parâmetro de Calvo nos salários & 0.75 \\
\hline$r_{\Delta \pi}$ & coeficiente do crescimento da inflação na regra de Taylor & 0.3 \\
\hline$r_{\pi}$ & coeficiente da inflação na regra de Taylor & 1.7 \\
\hline$r_{\Delta y}$ & coeficiente do crescimento do gap do produto na regra de Taylor & 0.0625 \\
\hline$r_{y}$ & coeficiente do gap do produto na regra de Taylor & 0.125 \\
\hline$\rho_{R}$ & Parâmetro de suavização da taxa de juros na regra de Taylor & 0.8 \\
\hline$\rho_{\bar{\pi}}$ & Parâmetro de suavização do choque na meta de inflação & 0.85 \\
\hline$\rho_{a}$ & Persistência do choque tecnológico & 0.85 \\
\hline$\rho_{b}$ & Persistência do choque de preferência & 0.85 \\
\hline$\rho_{E l}$ & Persistência do choque de oferta de trabalho & 0.85 \\
\hline$\rho_{w}$ & Persistência do choque no mark up dos salários & 0.85 \\
\hline$\rho_{g}$ & Persistência do choque fiscal & 0.85 \\
\hline$\rho_{y}$ & coeficiente do gap do produto na regra fiscal & 0.0 \\
\hline$\sigma_{R}$ & desvio padrão do choque da taxa de juros & 0.1 \\
\hline$\sigma_{\bar{\pi}}$ & desvio padrão do choque na meta de inflação & 0.2 \\
\hline$\sigma_{a}$ & desvio padrão do choque tecnológico & 0.4 \\
\hline$\sigma_{b}$ & desvio padrão do choque de preferência & 0.2 \\
\hline$\sigma_{E l}$ & desvio padrão do choque na oferta de trabalho & 0.1 \\
\hline$\sigma_{g}$ & desvio padrão do choque fiscal & 0.3 \\
\hline$\sigma_{w}$ & desvio padrão do choque no mark up do salário & 0.25 \\
\hline
\end{tabular}

Fonte: Elaboração própria 
Figura 1: IRF Bayesiana: Modelo com preços flexíveis.
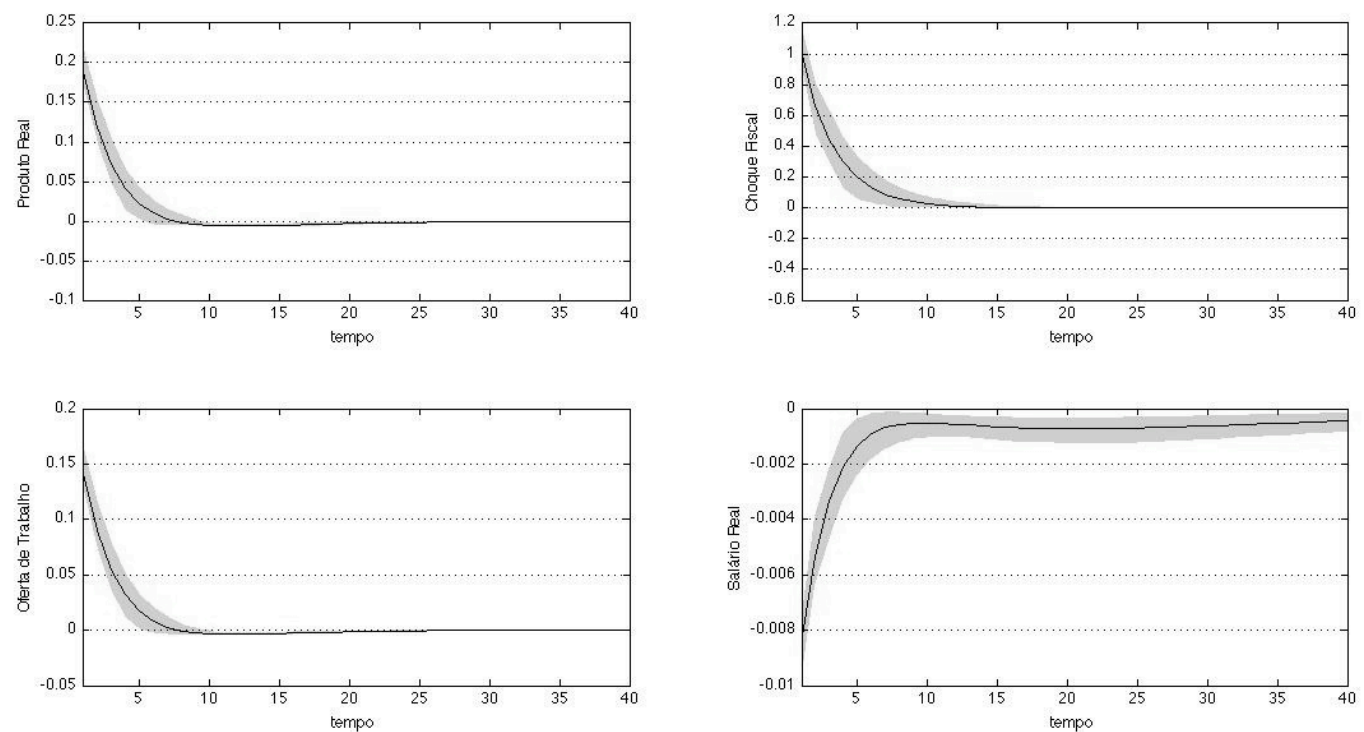

Nota: Linha sólida representa a mediana da resposta a um choque de 1\% nos Gastos do Governo. A área sombreada representa o intervalo de confiança de 68\%. Fonte: Elaboração Própria.

Figura 2: IRF Bayesiana: Modelo com preços rígidos.
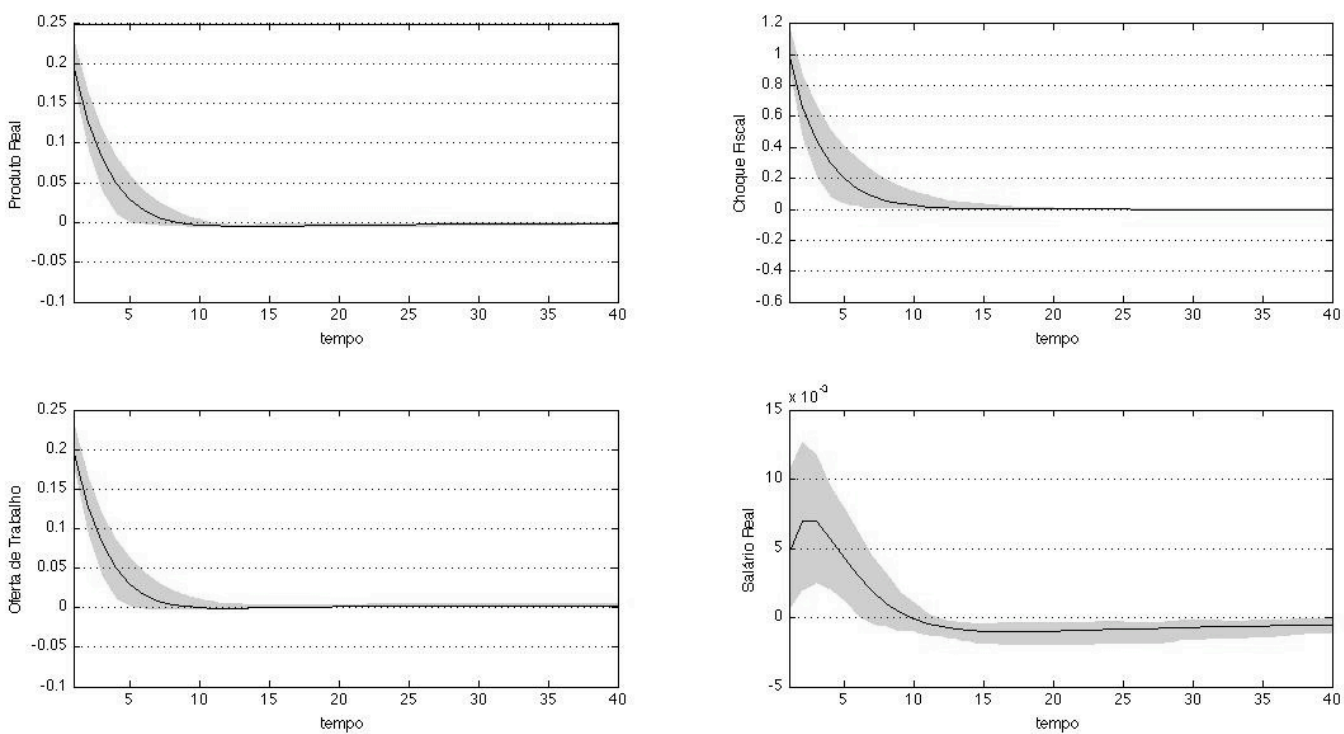

Nota: Linha sólida representa a mediana da resposta a um choque de $1 \%$ nos Gastos do Governo. A área sombreada representa o intervalo de confiança de 68\%. Fonte: Elaboração Própria. 
Tabela 2: Resultados da Estimação: Parâmetros

\begin{tabular}{lcllllll}
\hline \multirow{2}{*}{ Parâmetros } & $\begin{array}{l}\text { Distribuição } \\
\text { a priori }\end{array}$ & $\begin{array}{l}\text { Média a } \\
\text { Priori }\end{array}$ & $\begin{array}{l}\text { s.d. a } \\
\text { priori }\end{array}$ & $\begin{array}{l}\text { Média } \\
\text { Posterior }\end{array}$ & $\begin{array}{l}\text { Posterior } \\
\text { s.d. }\end{array}$ & HPD inf & HPD sup \\
\hline$\rho_{y}$ & unif & 0.000 & 1.0000 & -0.348 & 0.3557 & -0.8984 & 0.2852 \\
$\rho_{g}$ & beta & 0.850 & 0.1000 & 0.650 & 0.0994 & 0.4872 & 0.8055 \\
$\rho_{a}$ & beta & 0.850 & 0.1000 & 0.963 & 0.0215 & 0.9315 & 0.9964 \\
$\rho_{\bar{\pi}}$ & beta & 0.850 & 0.1000 & 0.951 & 0.0178 & 0.9197 & 0.9794 \\
$\rho_{b}$ & beta & 0.850 & 0.1000 & 0.480 & 0.0840 & 0.3584 & 0.6310 \\
$\rho_{l}$ & beta & 0.850 & 0.1000 & 0.930 & 0.0388 & 0.8714 & 0.9931 \\
$\sigma_{c}$ & norm & 1.000 & 0.3750 & 1.581 & 0.3662 & 0.9735 & 2.1848 \\
$\lambda$ & beta & 0.700 & 0.1000 & 0.963 & 0.0127 & 0.9446 & 0.9833 \\
$\xi_{w}$ & beta & 0.750 & 0.0500 & 0.668 & 0.0665 & 0.5622 & 0.7844 \\
$\xi_{p}$ & beta & 0.750 & 0.0500 & 0.685 & 0.0502 & 0.6065 & 0.7670 \\
$\gamma_{w}$ & beta & 0.750 & 0.1500 & 0.795 & 0.1004 & 0.6397 & 0.9664 \\
$\gamma_{p}$ & beta & 0.750 & 0.1500 & 0.401 & 0.1117 & 0.2183 & 0.5881 \\
$r_{\pi}$ & norm & 1.700 & 0.1000 & 1.346 & 0.0908 & 1.2001 & 1.4656 \\
$r_{\Delta \pi}$ & norm & 0.300 & 0.1000 & 0.563 & 0.0701 & 0.4508 & 0.6785 \\
$\rho$ & beta & 0.800 & 0.1000 & 0.476 & 0.0533 & 0.3822 & 0.5586 \\
$r_{y}$ & norm & 0.125 & 0.0500 & 0.176 & 0.0436 & 0.0982 & 0.2431 \\
$r_{\Delta y}$ & norm & 0.062 & 0.0500 & 0.103 & 0.0378 & 0.0500 & 0.1604 \\
\hline
\end{tabular}

Fonte: Elaboração própria

Tabela 3: Resultados da Estimação: desvio padrão dos choques

\begin{tabular}{lcllllll}
\hline \multirow{2}{*}{ Desvios } & $\begin{array}{l}\text { Distribuição } \\
\text { a priori }\end{array}$ & $\begin{array}{l}\text { Média a } \\
\text { Priori }\end{array}$ & $\begin{array}{l}\text { s.d. a } \\
\text { priori }\end{array}$ & $\begin{array}{l}\text { Média } \\
\text { Posterior }\end{array}$ & $\begin{array}{l}\text { Posterior } \\
\text { s.d. }\end{array}$ & HPD inf & HPD sup \\
\hline$\sigma_{a}$ & invg & 0.004 & 2.0000 & 0.011 & 0.0009 & 0.0092 & 0.0119 \\
$\sigma_{\bar{\pi}}$ & invg & 0.000 & 2.0000 & 0.228 & 0.0363 & 0.1709 & 0.2886 \\
$\sigma_{B}$ & invg & 0.002 & 2.0000 & 0.340 & 0.1002 & 0.1828 & 0.4992 \\
$\sigma_{E L}$ & invg & 0.001 & 2.0000 & 0.001 & 0.0011 & 0.0002 & 0.0018 \\
$\sigma_{R}$ & invg & 0.001 & 2.0000 & 0.320 & 0.0288 & 0.2764 & 0.3721 \\
$\sigma_{P}$ & invg & 0.008 & 2.0000 & 0.299 & 0.0344 & 0.2450 & 0.3569 \\
$\sigma_{W}$ & invg & 0.003 & 2.0000 & 0.001 & 0.0006 & 0.0006 & 0.0024 \\
$\sigma_{G}$ & invg & 0.003 & 2.0000 & 0.028 & 0.0030 & 0.0234 & 0.0330 \\
\hline
\end{tabular}

Fonte: Elaboração própria 
A intuição dos resultados acima é relativamente simples. Em economias com preços flexíveis e agentes ricardianos, o aumento dos gastos do governo tem um efeito riqueza negativo sobre as famílias, reduzindo o consumo e aumentando a oferta de trabalho. O que, dada a demanda por trabalho, implica em uma redução no salário real. Por outro lado, em economias com preços rígidos, um aumento nos gastos governamentais tem impacto direto na demanda agregada, afetando a demanda por trabalho, resultando assim em aumentos no produto e no emprego. O aumento da demanda por trabalho eleva o salário real. Vale salientar que as implicações do modelo DSGE estimado neste artigo são comuns tanto em modelos na tradição RBC quanto na tradição NK (Baxter e King (1993), Finn (1998) e Aiyagari et alii (1990)).

A partir das funções de impulso resposta é possível, portanto, derivar as restrições que serão utilizadas na identificação dos choques fiscais no modelo SVAR na próxima seção. Como é possível observar, sob a hipótese de preços flexíveis, choques fiscais aumentam contemporaneamente o produto, o emprego e reduz o salário real. Por outro lado, na economia com preços e salários rígidos, os efeitos contemporâneos de choques fiscais sobre o produto, trabalho e salário real são positivos. Portanto, em ambas as economias, tanto o produto quanto à oferta de trabalho aumentam em resposta a um choque fiscal. Serão estas, portanto, as restrições a serem impostas na estratégia empírica. A tabela a seguir resume os resultados.

Tabela 4: Resultados do modelo de equilíbrio geral

\begin{tabular}{ccccc}
\hline Modelo & Produto & Gastos do Governo & Emprego & Salário Real \\
\hline Preços Flexíveis & + & + & + & - \\
Preços Rígidos & + & + & + & + \\
\hline
\end{tabular}

Fonte: Elaboração própria

\section{ESTRATÉGIA EMPÍRICA}

\subsection{Dados}

Os dados utilizados nessa pesquisa, bem como seus períodos e suas respectivas fontes, são apresentados na Tabela 5 abaixo. Todos os dados são de periodicidade mensal, exceto pela série de consumo final da administração pública que possui periodicidade trimestral. ${ }^{3}$

\subsection{Modelo Empírico}

A relação dinâmica entre as variáveis de interesse pode ser representada através de um modelo Vetorial Autorregressivo Estrutural (SVAR) da seguinte maneira:

$$
\mathbf{y}_{\mathbf{t}}^{\top} \mathbf{A}_{\mathbf{0}}=\sum_{\ell=\mathbf{1}}^{\mathbf{p}} \mathbf{y}_{\mathbf{t}-\ell}^{\top} \mathbf{A}_{\ell}+\varepsilon_{\mathbf{t}}^{\top} \text { para } 1 \leq t \leq T,
$$

em que $\mathbf{y}_{\mathbf{t}}$ é um vetor coluna $n \times 1$ das variáveis endógenas do modelo, $\mathbf{A}_{\mathbf{0}}$ é uma matriz $n \times n$ dos coeficientes contemporâneos, $\mathbf{A}_{\mathbf{t}}$ é uma matriz $n \times n$ dos parâmetros das variáveis defasadas, $\varepsilon_{t}$ é um vetor $n \times 1$ dos distúrbios estruturais, $p$ é a ordem de defasagem e $T$ é o tamanho da amostra.

\footnotetext{
${ }^{3}$ A Pesquisa Mensal do Emprego do IBGE (PME/IBGE) passou por uma mudança metodológica em março de 2002. Então, as taxas de variação da antiga pesquisa foram utilizadas para recuperar os dados anteriores a 2002 na nova pesquisa.
} 
Tabela 5: Dados

\begin{tabular}{|c|c|c|c|}
\hline Variável & & Descrição & Período \\
\hline G & $\begin{array}{l}\text { Consumo Real } \\
\text { do Governo }\end{array}$ & $\begin{array}{l}\text { Despesas públicas em custeio e investimento deflacio- } \\
\text { nado pelo índice de preços ao consumidor (IPCA). Fonte: } \\
\text { Ministério da Fazenda, Secretaria do Tesouro Nacional. }\end{array}$ & $\begin{array}{l}\text { Jan/1995- } \\
\text { Nov/2012 }\end{array}$ \\
\hline $\mathrm{Y}$ & PIB Real & $\begin{array}{l}\text { PIB do Brasil deflacionado pelo índice de preços ao con- } \\
\text { sumidor (IPCA). Fonte: Banco Central do Brasil. }\end{array}$ & $\begin{array}{l}\text { Jan/1995- } \\
\text { Nov/2012 }\end{array}$ \\
\hline $\mathrm{N}$ & Emprego & $\begin{array}{l}\text { Pessoas de } 10 \text { anos ou mais de idade, ocupadas na se- } \\
\text { mana de referência (mil pessoas). Fonte: IBGE - Pes- } \\
\text { quisa Mensal do Emprego (PME). }\end{array}$ & $\begin{array}{l}\text { Jan/1995- } \\
\text { Nov/2012 }\end{array}$ \\
\hline W & Salário Real & $\begin{array}{l}\text { Rendimento médio real do trabalho principal, efetiva- } \\
\text { mente recebido no mês de referência, pelas pessoas de } \\
10 \text { anos ou mais de idade, ocupadas na semana de refe- } \\
\text { rência (RS). Fonte: IBGE - Pesquisa Mensal do Emprego } \\
\text { (PME). }\end{array}$ & $\begin{array}{l}\text { Jan/1995- } \\
\text { Nov/2012 }\end{array}$ \\
\hline $\mathrm{H}$ & $\begin{array}{l}\text { Horas Trabalha- } \\
\text { das }\end{array}$ & $\begin{array}{l}\text { Número total de horas pagas ao pessoal ocupado assala- } \\
\text { riado (número índice). Fonte: IBGE - Pesquisa Industrial } \\
\text { Mensal do Emprego e Salário (PIMES). }\end{array}$ & $\begin{array}{l}\text { Jan/1995- } \\
\text { Nov/2012 }\end{array}$ \\
\hline G2 & $\begin{array}{l}\text { Consumo Real } \\
\text { do Governo }\end{array}$ & $\begin{array}{l}\text { Consumo final da administração pública deflacionado } \\
\text { pelo índice de preços ao consumidor (IPCA). Fonte: IBGE } \\
\text { - Sistema de Contas Nacionais. }\end{array}$ & $\begin{array}{l}\text { 1Trim./1995- } \\
\text { 3Trim./ } 2012\end{array}$ \\
\hline
\end{tabular}

Fonte:Elaboração própria.

A distribuição de $\varepsilon_{t}$, condicional as informações passadas, é Gaussiana com média e matriz de variância-covariância dada por, respectivamente, $E\left(\varepsilon_{t} \mid \mathbf{y}_{\mathbf{t}}, \ldots, \mathbf{y}_{\mathbf{t}-\ell}\right)$ e $E\left(\varepsilon_{t} \varepsilon_{t-1}^{\top} \mid \mathbf{y}_{\mathbf{1}}, \ldots, \mathbf{y}_{\mathbf{t}-\ell}\right)=$ $I_{n \times n}$. Pós-multiplicando todos os elementos de (4) por $\mathbf{A}_{0}^{-1}$, obtêm-se o Modelo Vetorial Autorregressivo em sua forma reduzida:

$$
\mathbf{y}_{\mathbf{t}}^{\top}=\sum_{\ell=1}^{\mathbf{p}} \mathbf{y}_{\mathbf{t}-\ell}^{\top} \mathbf{B}_{\ell}+\mathbf{u}_{\mathbf{t}}^{\top} \text { para } 1 \leq t \leq T
$$

em que $\mathbf{B}_{\mathbf{t}}=\mathbf{A}_{\ell} \mathbf{A}_{\mathbf{0}}^{-\mathbf{1}}$ (para $\left.\ell=1,2, \ldots, p\right)$, $\mathbf{u}_{\mathbf{t}}^{\top}=\boldsymbol{\varepsilon}_{\mathbf{t}}^{\top} \mathbf{A}_{\mathbf{0}}^{-\mathbf{1}}$ e $E\left[\mathbf{u}_{\mathbf{t}}^{\top} \mathbf{u}_{\mathbf{t}}\right]=\mathbf{\Omega}=\left(\mathbf{A}_{\mathbf{0}}^{\top} \mathbf{A}_{\mathbf{0}}\right)^{-\mathbf{1}}$ é a matriz de variância-covariância dos resíduos na forma reduzida.

Neste trabalho, o vetor $\mathbf{y}_{t}$ é composto pelo logaritmo natural de uma medida de gastos do governo, uma medida de produto agregado nacional, uma medida do nível de emprego e uma medida do nível do salário real.

Quatro modelos foram estimados usando diferentes combinações das séries de dados. O objetivo com isto foi verificar se os resultados dependeriam do conceito da variável utilizada no modelo empírico. A Tabela 6, a seguir, organiza as especificações utilizadas. Em todos os modelos, as variáveis foram dessazonalizadas, colocadas em escala logarítmica, e tiveram tendência e média removidas. No caso dos modelos que usam dados trimestrais, modelos 3 e 4, as séries de PIB, emprego e salário são dadas pela média a cada três meses.

A ordem de defasagem de cada um dos modelos VAR em sua forma reduzida foi determinada pelo critério de seleção de Schwarz (critério de seleção bayesiano) para os modelos com séries de periodicidade trimestral e pelo critério de seleção de Akaike para os modelos com séries de periodicidade 
Tabela 6: Especificações dos Modelos

\begin{tabular}{clllll}
\hline Modelo & \multicolumn{3}{r}{ Especificação } & Periodicidade dos Dados \\
\hline 1 & $\mathbf{y}_{\mathbf{t}}=\left[\begin{array}{lllll}G_{t} & Y_{t} & N_{t} & W_{t}\end{array}\right]$ & Mensal \\
2 & $\mathbf{y}_{\mathbf{t}}=\left[\begin{array}{lllll}G_{t} & Y_{t} & H_{t} & W_{t}\end{array}\right]$ & Mensal \\
3 & $\mathbf{y}_{\mathbf{t}}=\left[\begin{array}{lllll}G 2_{t} & Y_{t} & N_{t} & W_{t}\end{array}\right]$ & Trimestral \\
4 & $\mathbf{y}_{\mathbf{t}}=\left[\begin{array}{lllll}G 2_{t} & Y_{t} & H_{t} & W_{t}\end{array}\right]$ & Trimestral \\
\hline
\end{tabular}

Fonte: Elaboração própria.

mensal, assim como sugerido por Ivanov e Kilian (2005). Além disto, a definição da ordem de defasagem considerou os resultados do teste de autocorrelação (estatística $Q$ multivariada). Tomando por base os resultados desse procedimento, os modelos foram estimados com duas defasagens em todas as especificações (os resultados dos testes são apresentados no Apêndice).

Muito embora o modelo a ser estimado consista no VAR na sua forma reduzida, as questões de interesse deste trabalho demandam a obtenção de funções de resposta a choques estruturais. Essas funções representam as respostas endógenas das variáveis do sistema a um impulso em elementos do vetor $\varepsilon_{t}$. Na identificação de choques nos gastos do governo, foi adotada uma estratégia de restrição de sinais, a qual é descrita abaixo.

\subsection{Estratégia de Identificação}

Nesse trabalho, utiliza-se a estratégia de identificação de restrições de sinal proposta por Uhlig (2005). No entanto, seguimos Canova (2002), Dedola e Neri (2007) e Pappa (2009), buscando as características de um choque fiscal em um modelo DSGE - como na Seção 2 - e aplicando o método de restrição de sinal pura a partir dessas características.

O problema de identificação consiste em obter uma matriz $\mathbf{A}_{\mathbf{0}}$ que relacione o erro na forma reduzida, $\mathbf{u}_{\mathbf{t}}$, ao choque estrutural, $\varepsilon_{t}$. Essa matriz está relacionada à matriz de variância e covariância dos resíduos do VAR na forma reduzida, $\Omega$, da seguinte forma:

$$
\boldsymbol{\Omega}=\left(\mathbf{A}_{\mathbf{0}}^{\top} \mathbf{A}_{\mathbf{0}}\right)^{-\mathbf{1}} .
$$

Admitindo que $\Omega=\tilde{\mathbf{A}} \tilde{\mathbf{A}}^{\top}$, onde $\tilde{\mathbf{A}}$ é uma matriz de decomposição de Choleski, então,

$$
\mathbf{A}_{\mathbf{0}}^{-1}=\tilde{\mathbf{A}}^{\top} \mathbf{Q}
$$

onde $\mathbf{Q}$ é uma matriz ortogonal, ou seja, $\mathbf{Q}^{\top} \mathbf{Q}=\mathbf{I}_{\mathbf{n} \times \mathbf{n}}$. Dessa forma temos que qualquer vetor de impulso pode ser escrito como:

$$
\mathbf{a}=\tilde{\mathbf{A}} \boldsymbol{\alpha},
$$

onde, a é uma coluna de $\mathbf{A}_{\mathbf{0}}^{-1}$ que contém as respostas contemporâneas das variáveis a um determinado choque, e $\boldsymbol{\alpha}$ é uma coluna de $\mathbf{Q}$ na posição correspondente. Assim, os choques de um impulso a $\left(r_{a}\right)$ no período $k$ podem ser calculados da seguinte forma:

$$
r_{a}(k)=\sum_{i=1}^{n} \alpha_{i} r_{i}(k),
$$

onde, $r_{i}(k)$ são as respostas da i-ésima variável aos choques da decomposição de Choleski no período $k$, e $\alpha_{i}$ é o valor do elemento do vetor $\alpha$ na posição $i$. 
Com base na equação acima, é possível determinar as respostas das variáveis a um choque fiscal. É preciso, para tanto, definir o choque fiscal. Nesse sentido, restringimos as respostas conforme os resultados apresentados na Tabela 4, e formulamos a seguinte suposição:

Suposição 1 (Vetor de choque fiscal). Um vetor-impulso de política fiscal é um vetor-impulso a, tal que as impulso-respostas $r_{a}(k)$ dos gastos do governo, do produto e do emprego (horas trabalhadas) sejam nãonegativas nos períodos $k=0, \ldots, K$.

Neste estudo, consideramos o período de um semestre no qual as restrições sobre as respostas devem se manter. A justificativa para a imposição de $k=6$ nos modelos mensais e $k=2$ nos modelos trimestrais pode ser encontrada no modelo DSGE estimado, onde as variáveis em questão respondem por mais de dois trimestres a choques fiscais. Portanto, impondo restrições de dois trimestres não violaria as restrições derivadas a partir do modelo DSGE. ${ }^{4}$

A definição do choque fiscal não é suficiente para garantir a identificação exata do choque, porque dadas uma matriz de coeficientes do VAR na forma reduzida, $\mathbf{B}$, uma matriz de variância e covariância dos resíduos, $\Omega$, e um horizonte de tempo para a restrição de sinal, $K$, o conjunto de vetores-impulso que atendem a suposição $1, \mathcal{A}(\mathbf{B}, \Omega, K)$, pode conter muitos elementos. Segundo Uhlig (2005), isso ocorre porque os vetores-impulso são obtidos por meio de restrições de desigualdade.

Para obter a identificação exata dos choques fiscais, utilizou-se uma abordagem bayesiana, na qual os parâmetros do modelo VAR na forma reduzida, $\mathbf{B}$ e $\boldsymbol{\Omega}$, podem apresentar realizações que geram respostas aos choques que infringem ou não os movimentos esperados pela teoria econômica.

Para selecionar as realizações que estão de acordo com a teoria econômica, impomos que $\mathbf{B}$ segue uma distribuição conjunta Normal-Wishart, com média e variância determinadas pelos valores obtidos na estimação por mínimos quadrados ordinários. Então, extraímos 200 valores para $\mathbf{B}$ dessa distribuição a priori. Em seguida, para cada um desses conjuntos de parâmetros, retiramos aleatoriamente 200 valores dos choques iniciais dentro de um esfera unitária com dimensão igual a 4 (i.e. o número de variáveis do sistema), totalizando um conjunto de 40.000 impulsos, dos quais selecionamos 10.000 que atendem à Suposição 1. É importante ressaltar que, no caso dos choques iniciais que não coadunavam com a restrição, os sinais dos mesmos foram invertidos assim como sugerido por Uhlig (2005).

Por fim, considerando os 10.000 choques selecionados, calculamos a distribuição a posteriori das respostas. Com base nessa distribuição, construímos e analisamos as funções de impulso-resposta.

De acordo com Pappa (2009), além de aproximar o modelo teórico aos dados, a utilização de restrições de sinal robustas evita problemas típicos associados com a identificação de choques fiscais com significado econômico. Em particular, as restrições de sinal solucionam a falta de argumentos para um número suficiente de restrições de choques iguais à zero, como enfatizado por Mountford e Uhlig (2009), Canova e Pappa (2007) e Perotti (2004).

Além disso, ainda segundo Pappa (2009), os problemas envolvendo a endogeneidade das variáveis fiscais e a defasagem entre planejamento, aprovação e execução das políticas fiscais são contornados. Isso porque todas as variáveis relevantes são endógenas, a teoria define as características dos choques fiscais de interesse, e não há restrição para o tempo de resposta das variáveis.

\section{RESULTADOS}

Nessa seção, são discutidas as evidências, encontradas para o Brasil, dos efeitos de choques fiscais sobre variáveis de mercado de trabalho com base na abordagem SVAR com restrições de sinais. Como discutido na seção anterior, foram estimados quatro tipos de modelos SVAR, onde a diferença entre os modelos consistiu na definição das variáveis utilizadas. As funções impulso-resposta de cada um dos

\footnotetext{
${ }^{4}$ Outro exercício para avaliar a robustez dos resultados consistiu em alterar o período de restrição para um trimestre nos casos dos modelos 1 e 2 (dados mensais).
} 
modelos estimados são apresentadas nas figuras a seguir. Em todos os casos, as linhas tracejadas representam o intervalo de confiança de $68 \%$ e a linha sólida representa a resposta mediana das respostas.

De uma forma geral, em todos os modelos estimados, choques fiscais apresentaram um impacto positivo sobre o produto real, sobre a oferta de trabalho e sobre os salários reais, em consonância, portanto, com as implicações advindas de uma economia com rigidez de preços e salários.

Em particular, os resultados do modelo 1 (Figura 3) indicam que o efeito de um choque que aumenta os gastos do governo em $1 \%$, tende a apresentar um efeito positivo sobre o nível de atividade econômica. O PIB apresenta crescimento contemporâneo de 0,08\%. Esse efeito positivo é prolongado, mas tende a se dissipar no longo prazo.

Esse resultado vai de encontro aquele encontrado por Mendonça et alii (2010), em que o produto tem maior probabilidade de sofrer um impacto negativo dado um choque fiscal. No entanto, Peres e Ellery Jr (2009) também encontram efeitos positivos de choques fiscais sobre o produto, porém pouco persistentes.

O comportamento do nível de emprego é similar nos primeiros meses. O choque tem efeito positivo sobre essa variável durante quase 18 meses (um ano e meio). 0 choque inicial é da ordem de $0,01 \%$, e o efeito acumulado após 12 meses é de $0,12 \%$.

Figura 3: Respostas a choques nos gastos do governo - Modelo 1
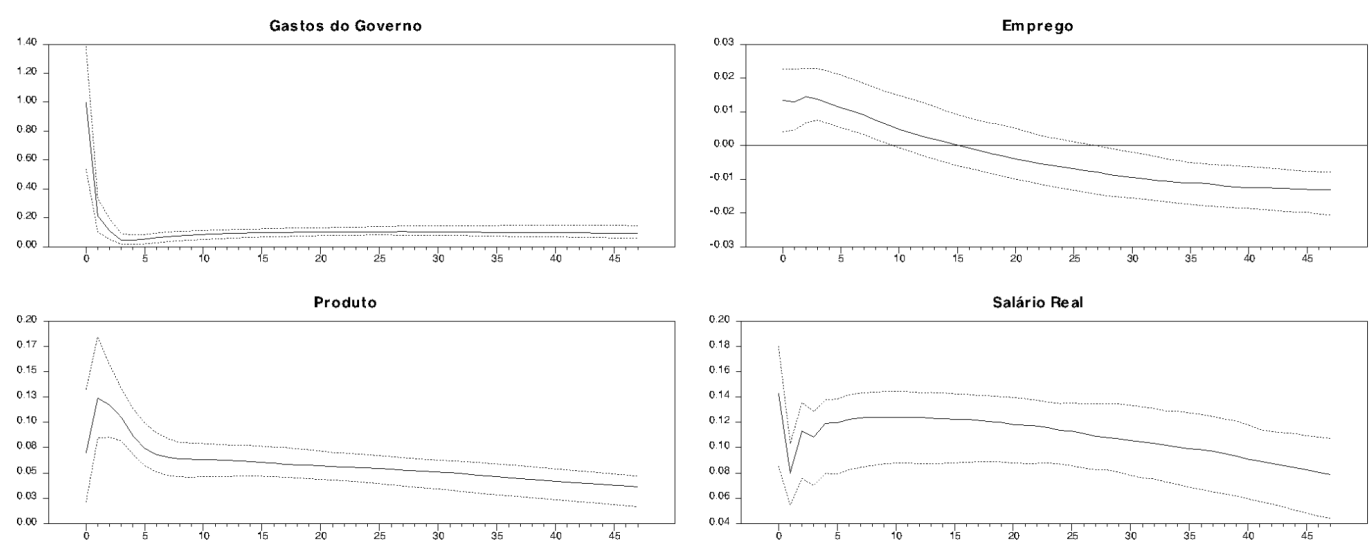

Choque Fiscal - Modelo 1

Fonte: Elaboração Própria. Função impulso-resposta das variáveis do modelo 1. O eixo horizontal representa os meses após o choque. As linhas tracejadas indicam o intervalo de confiança de $68 \%$ e as linhas sólidas, as medianas.

Assim como no caso do emprego, o impacto do choque fiscal sobre o salário real é positivo. A resposta da variável é positiva durante todo período examinado. 0 valor máximo da resposta é alcançado no mês contemporâneo ao choque, o que mostra uma rápida reação dessa variável. No acumulado de 12 meses, o salário real aumenta em $1,2 \%$.

No modelo 2 (Figura 4), que foi estimado considerando as horas trabalhadas ao invés do nível de emprego, os resultados são semelhantes em termos da dinâmica das variáveis ao longo do período estudado. Novamente, o choque fiscal produziu efeitos positivos e prolongados nas variáveis relacionadas ao mercado de trabalho.

No caso do modelo 3 (Figura 5), que considera dados trimestrais e uma definição alternativa de gastos governamentais, os resultados apresentam algumas pequenas mudanças. A dinâmica do produto permanece caracterizada por respostas positivas e uma tendência de se dissipar no longo prazo. $\mathrm{O}$ em- 
Figura 4: Respostas a choques nos gastos do governo - Modelo 2
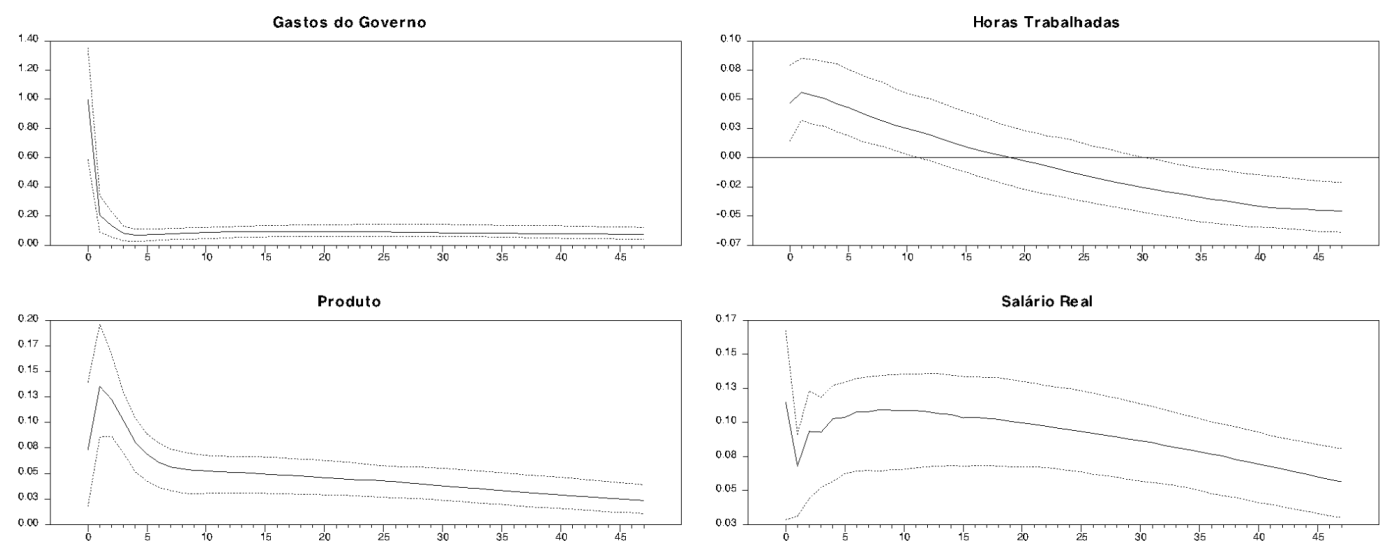

Choque Fiscal - Modelo 2

Fonte: Elaboração Própria. Função impulso-resposta das variáveis do modelo 2. 0 eixo horizontal representa os meses após o choque. As linhas tracejadas indicam o intervalo de confiança de $68 \%$ e as linhas sólidas, as medianas.

prego cresce até o oitavo trimestre, e o efeito de maior magnitude é alcançado no segundo trimestre após o choque inicial. $\mathrm{O}$ salário real apresenta uma elevada probabilidade de responder contemporaneamente de modo positivo ao choque fiscal. Ao contrário dos modelos anteriores, o efeito positivo tende a aumentar, atingindo a resposta máxima por volta do nono trimestre.

Figura 5: Respostas a choques nos gastos do governo - Modelo 3
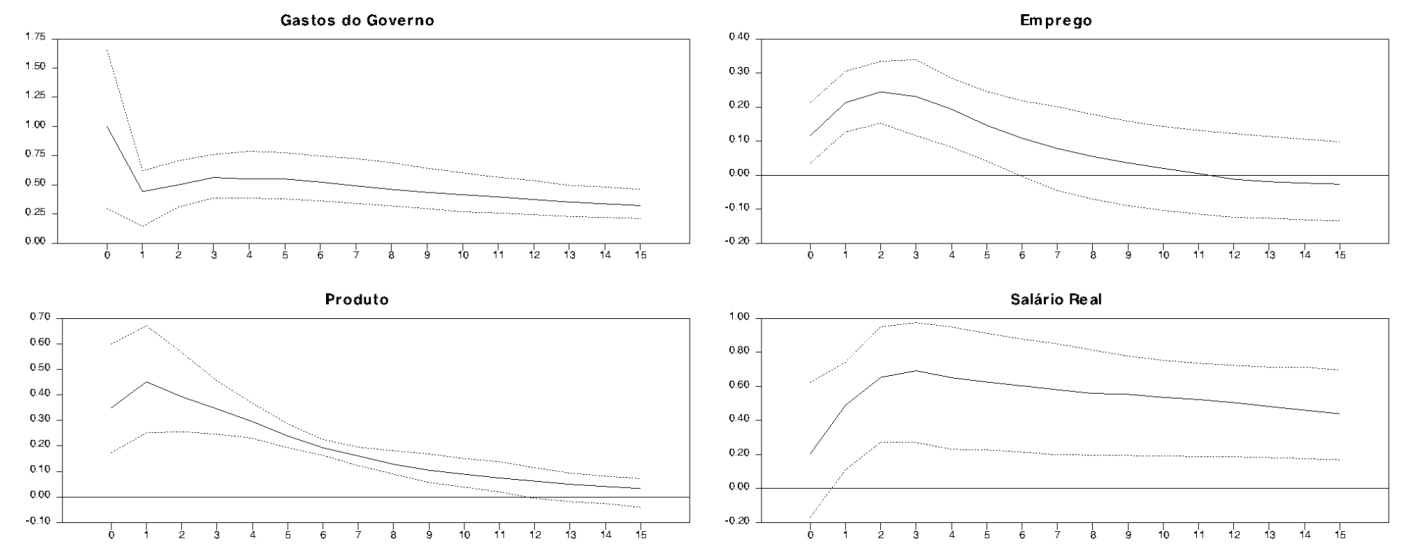

Choque Fiscal - Modelo 3

Fonte: Elaboração Própria. Função impulso-resposta das variáveis do modelo 3. O eixo horizontal representa os meses após o choque. As linhas tracejadas indicam o intervalo de confiança de $68 \%$ e as linhas sólidas, as medianas. 
O modelo 4 foi estimado considerando as horas trabalhadas ao invés do nível de emprego e dados de periodicidade trimestral. O resultado da estimação desse modelo (Figura 6) é bastante semelhante ao do modelo 3. Mais uma vez, o choque fiscal produziu efeitos positivos e prolongados nas variáveis relacionadas ao mercado de trabalho.

Figura 6: Respostas a choques nos gastos do governo - Modelo 4
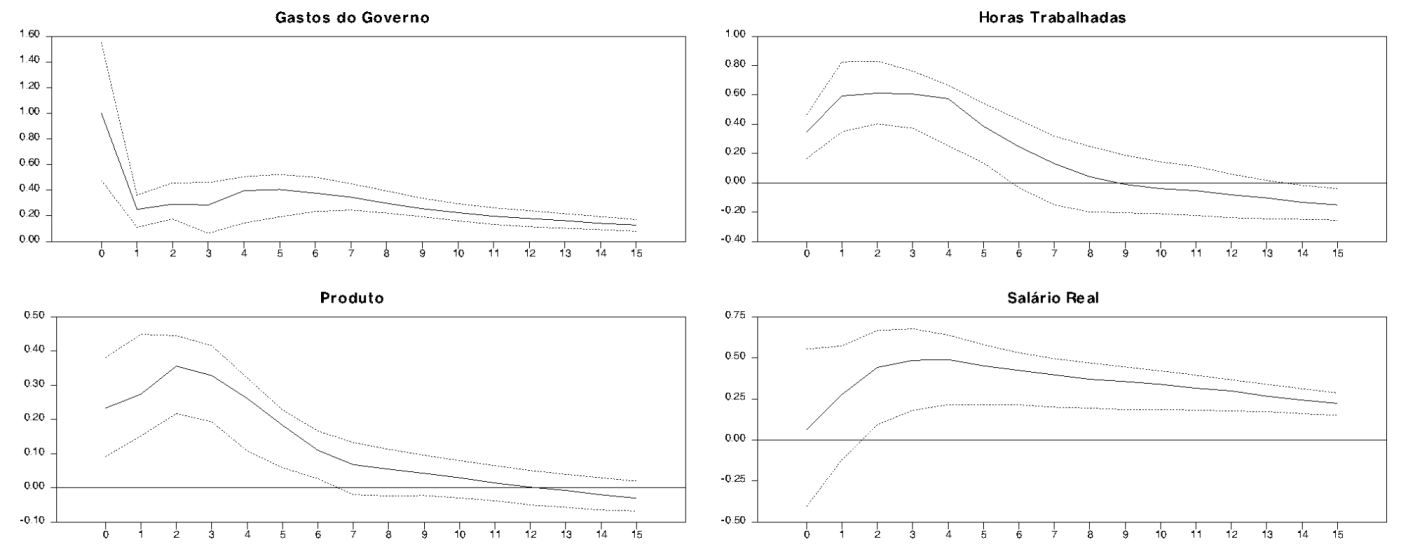

Choque Fiscal - Modelo 4

Fonte: Elaboração Própria. Função impulso-resposta das variáveis do modelo 4. O eixo horizontal representa os meses após o choque. As linhas tracejadas indicam o intervalo de confiança de $68 \%$ e as linhas sólidas, as medianas.

Em resumo, as funções de resposta a impulso estimadas com base nos diferentes modelos SVAR estimados mostram evidências de que choques nos gastos governamentais apresentam impactos positivos sobre o produto, o trabalho e o salário real.

A esta altura vale a pena comparar os resultados obtidos neste artigo com trabalhos similares. Em particular, utilizando a mesma abordagem utilizada neste artigo (modelo DSGE mais modelo SVAR com restrição de sinal), Pappa (2009) também encontrou evidências de que choques nos gastos governamentais elevam o produto, o emprego e o salário real nos Estados Unidos. De igual maneira, a evidência com base apenas em modelos DSGE (estimados ou não), na maior parte dos casos, é de que choques fiscais apresentam um impacto positivo sobre o emprego e sobre o salário real. Estes são os casos, por exemplo, dos modelos DSGE de Galí et alii (2007), Coenen e Straub (2005) em um modelo DSGE estimado para a zona do Euro, e Gadelha e Divino (2012) em um modelo DSGE estimado para o Brasil. No entanto, este resultado vai de encontro ao obtido por Silva e Portugal (2010), onde o efeito sobre salário real de um choque nos gastos do governo é negativo e persistente.

\section{ANÁLISE DE ROBUSTEZ}

De maneira a avaliar a robustez dos resultados discutidos na seção anterior, os modelos foram estimados novamente considerando algumas modificações. Primeiramente, todos os modelos foram estimados considerando um período de três meses para as restrições. Posteriormente, esse período foi modificado para um mês nos casos dos modelos 1 e 2 . Os gráficos das funções de impulso resposta dessas novas estimações encontram-se no apêndice (Figuras B-1 e B-2). De uma forma geral, nos dois exercícios, os resultados não apresentaram mudanças significativas em relação àqueles apresentados anteriormente, ou seja, seguindo um choque fiscal o produto, o emprego e o salário real aumentam. 
Portanto, os resultados de que choques fiscais elevam o emprego e o salário real e, portanto, têm caráter Novo Keynesiano no Brasil, foram consistentes a mudanças no período das restrições de sinal.

\section{CONSIDERAÇÕES FINAIS}

Esse trabalho encontrou evidências empíricas de como variáveis do mercado de trabalho brasileiro se comportam em resposta a choques fiscais. O método empírico adotado foi o de Vetores Autoregressivos Estruturais e a estratégia de identificação seguiu a metodologia de validação de modelos DSGE proposta por Canova (2002).

Em particular, este estudo utilizou as implicações de choques fiscais sobre uma série de variáveis macroeconômicas a partir de um modelo DSGE na tradição da Nova Síntese Neoclássica estimado utilizando métodos de inferência Bayesiana para o Brasil. O modelo DSGE estimado produziu respostas positivas do produto e do emprego a elevações (choques positivos) nos gastos do governo. Por outro lado, os efeitos sobre os salários reais foram dependentes de qual hipótese sobre a flexibilidade de preços foi assumida. Na economia com preços flexíveis, o choque fiscal produziu uma redução no salário real, enquanto na economia com preços rígidos, o mesmo choque produziu elevações no salário real. De posse das restrições comuns às duas economias (preços flexíveis e preços rígidos), foi utilizado o método de identificação por restrição de sinais proposta por Uhlig (2005).

Os resultados mostraram que tanto o emprego quanto o salário real aumentam em resposta a um choque fiscal. Estes resultados sugerem, portanto, que choques fiscais deslocam a demanda por trabalho, elevando o emprego e o salário real na economia brasileira.

\section{BIBLIOGRAFIA}

Aiyagari, S. R., Christiano, L. J., \& Eichenbaum, M. (1990). The output, employment, and interest rate effects of government consumption. Working Paper 25, Federal Reserve Bank of Minneapolis.

Baxter, M. \& King, R. G. (1993). Fiscal policy in general equilibrium. American Economic Review, 83(3):315334.

Blanchard, O. \& Perotti, R. (2002). An Empirical Characterization of the Dynamic Effects of Changes in Government Spending and Taxes on Output. Quarterly Journal of Economics, 117(4):1329-1368.

Brooks, S. P. \& Gelman, A. (1998). General methods for monitoring convergence of iterative simulations. Journal of Computational and Graphical Statistics, 7:434-455.

Burnside, C., Eichenbaum, M., \& Fisher, J. D. M. (2004). Fiscal Shocks and their Consequences. Journal of Economic Theory, 115(1):89-117.

Calvo, G. A. (1983). Staggered prices in a utility-maximizing framework. Journal of Monetary Economics, 12(3):383-398.

Canova, F. (2002). Validating monetary DSGE models through VARs. CEPR Discussion Papers 3442, C.E.P.R. Discussion Papers.

Canova, F. \& Pappa, E. (2007). Price Differentials in Monetary Unions: The Role of Fiscal Shocks. The Economic Journal, 117(520):713-737.

Castro, M. R. D., Gouvea, S. N., Minella, A., dos Santos, R. C., \& Souza-Sobrinho, N. F. (2011). Samba: Stochastic analytical model with a bayesian approach. Working Papers Series 239, Central Bank of Brazil, Research Department. 
Coenen, G. \& Straub, R. (2005). Does government spending crowd in private consumption? Theory and empirical evidence for the Euro area. Working Paper Series 513, European Central Bank.

Dedola, L. \& Neri, S. (2007). What does a Technology Shock do? A VAR analysis with Model-Based Sign Restrictions. Journal of Monetary Economics, 54(2):512-549.

Fatás, A. \& Mihov, I. (2001). The Effects of Fiscal Policy on Consumption and Employment: Theory and Evidence. CEPR Discussion Papers 2760, C.E.P.R. Discussion Papers.

Finn, M. G. (1998). Cyclical Effects of Government's Employment and Goods Purchases. International Economic Review, 39(3):635-657.

Frankel, J. A., Végh, C. A., \& Vuletin, G. (2011). On Graduation from Fiscal Procyclicality. Working Paper 17619, National Bureau of Economic Research.

Gadelha, S. R. D. B. \& Divino, J. A. (2012). Estímulo fiscal, impostos distorcivos e ciclo econômico brasileiro. In Anais do 34 Encontro Brasileiro de Econometria.

Galí, J., López-Salido, J. D., \& Vallés, J. (2007). Understanding the effects of government spending on consumption. Journal of the European Economic Association, 5(1):227-270.

Ivanov, V. \& Kilian, L. (2005). A practitioner's guide to lag order selection for VAR impulse response analysis. Studies in Nonlinear Dynamics \& Econometrics, 9(1):2.

Mendonça, M. J. C., Medrano, L., \& Sachsida, A. (2010). Efeitos da política monetária na economia brasileira: Resultados de um procedimento de identificação agnóstica. Pesquisa e Planejamento Econômico, 40(3).

Mountford, A. \& Uhlig, H. (2009). What are the Effects of Fiscal Policy Shocks? Journal of Applied Econometrics, 24(6):960-992.

Pappa, E. (2009). The Effects Of Fiscal Shocks On Employment And The Real Wage. International Economic Review, 50(1):217-244.

Peres, M. A. F. \& Ellery Jr, R. G. E. (2009). Efeitos dinâmicos dos choques fiscais do governo central no PIB do Brasil. Pesquisa e Planejamento Econômico, 39(2).

Perotti, R. (2004). Estimating the effects of fiscal policy in OECD countries. Working Papers 276, IGIER (Innocenzo Gasparini Institute for Economic Research), Bocconi University.

Perotti, R. (2007). In Search of the Transmission Mechanism of Fiscal Policy. NBER Working Papers 13143, National Bureau of Economic Research, Inc.

Ramey, V. A. \& Shapiro, M. D. (1998). Costly Capital Reallocation and the Effects of Government Spending. Carnegie-Rochester Conference Series on Public Policy, 48(1):145-194.

Silva, F. S. \& Portugal, M. S. (2010). O Impacto de Choques Fiscais na Economia Brasileira: Uma Abordagem DSGE. Anais do $32^{\circ}$ Encontro Brasileiro de Econometria.

Sims, C. A. (2002). Solving Linear Rational Expectations Models. Computational Economics, 20(1-2):1-20.

Smets, F. \& Wouters, R. (2003). An estimated dynamic stochastic general equilibrium model of the Euro area. Journal of the European Economic Association, 1(5):1123-1175.

Smets, F. \& Wouters, R. (2007). Shocks and frictions in us business cycles: A bayesian DSGE approach. American Economic Review, 97(3):586-606.

Uhlig, H. (2005). What are the Effects of Monetary Policy on Output? Results from an Agnostic Identification Procedure. Journal of Monetary Economics, 52(2):381-419. 


\section{A. TESTES DE ESPECIFICAÇÃO DO MODELO EMPÍRICO}

Tabela A-1: Critérios de Seleção do Número de Defasagens

\begin{tabular}{ccccc}
\hline Defasagens & $\begin{array}{c}\text { Modelo 1 } \\
(\text { AIC })\end{array}$ & $\begin{array}{c}\text { Modelo 2 } \\
(\text { AIC })\end{array}$ & $\begin{array}{c}\text { Modelo 3 } \\
(\mathrm{BIC})\end{array}$ & $\begin{array}{c}\text { Modelo 4 } \\
(\mathrm{BIC})\end{array}$ \\
\hline 1 & 3753,00278 & 4158,11039 & 989,16465 & 1082,02451 \\
2 & 3722,86396 & 4099,52410 & 1018,94887 & 1095,67459 \\
3 & 3735,16449 & 4113,65505 & 1066,87209 & 1146,52465 \\
4 & 3762,66812 & 4141,25534 & 1091,83222 & 1177,72369 \\
5 & 3770,30230 & 4146,87469 & & \\
6 & 3790,50687 & 4182,68304 & & \\
\hline
\end{tabular}

Fonte: Elaboração própria

Tabela A-2: Testes de Autocorrelação e Normalidade dos Resíduos dos Modelos

\begin{tabular}{ccccc}
\hline Modelo & Ljung-Box (1) & Ljung-Box (2) & Ljung-Box (3) & Jarque-Bera \\
\hline \multirow{2}{*}{1} & 2,85606 & 14,43803 & 29,09076 & 903,448 \\
& $(0,99988)$ & $(0,99677)$ & $(0,98588)$ & $(0,000)$ \\
2 & 2,65886 & 15,8639 & 26,43903 & 1519.619 \\
& $(0,99993)$ & $(0,99236)$ & $(0,99516)$ & $(0,000)$ \\
3 & 1,74149 & 11,60105 & 31,27812 & 24,562 \\
& $(0,9999$ & $(0,99964)$ & $(0,97050)$ & $(0,002)$ \\
4 & 4,57723 & 14,38960 & 29,02994 & 35,235 \\
& $(0,99749)$ & $(0,99687)$ & $(0,98619)$ & $(0,000)$ \\
\hline
\end{tabular}

Fonte: Elaboração própria

\section{B. ANÁLISE DE ROBUSTEZ}




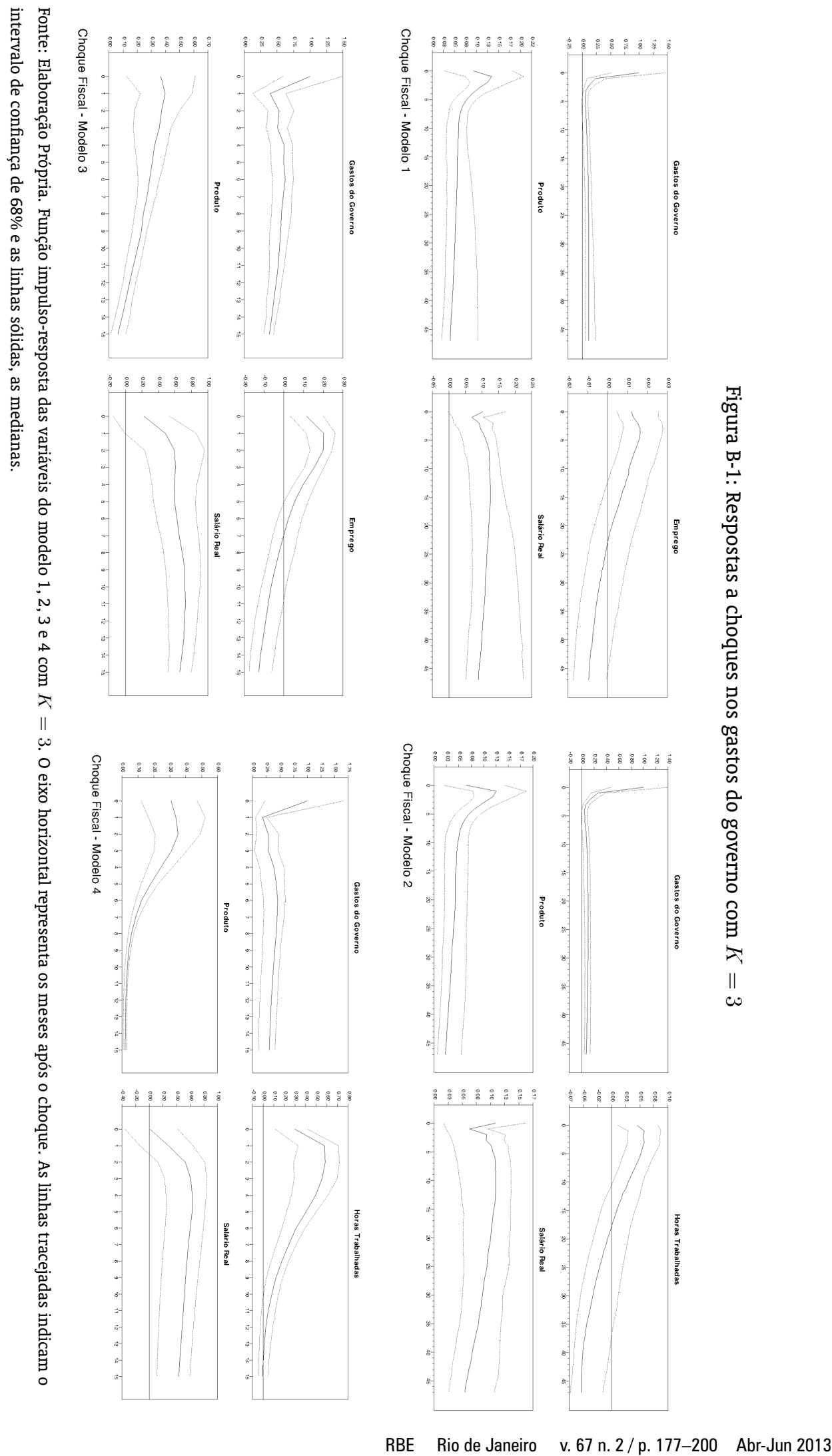




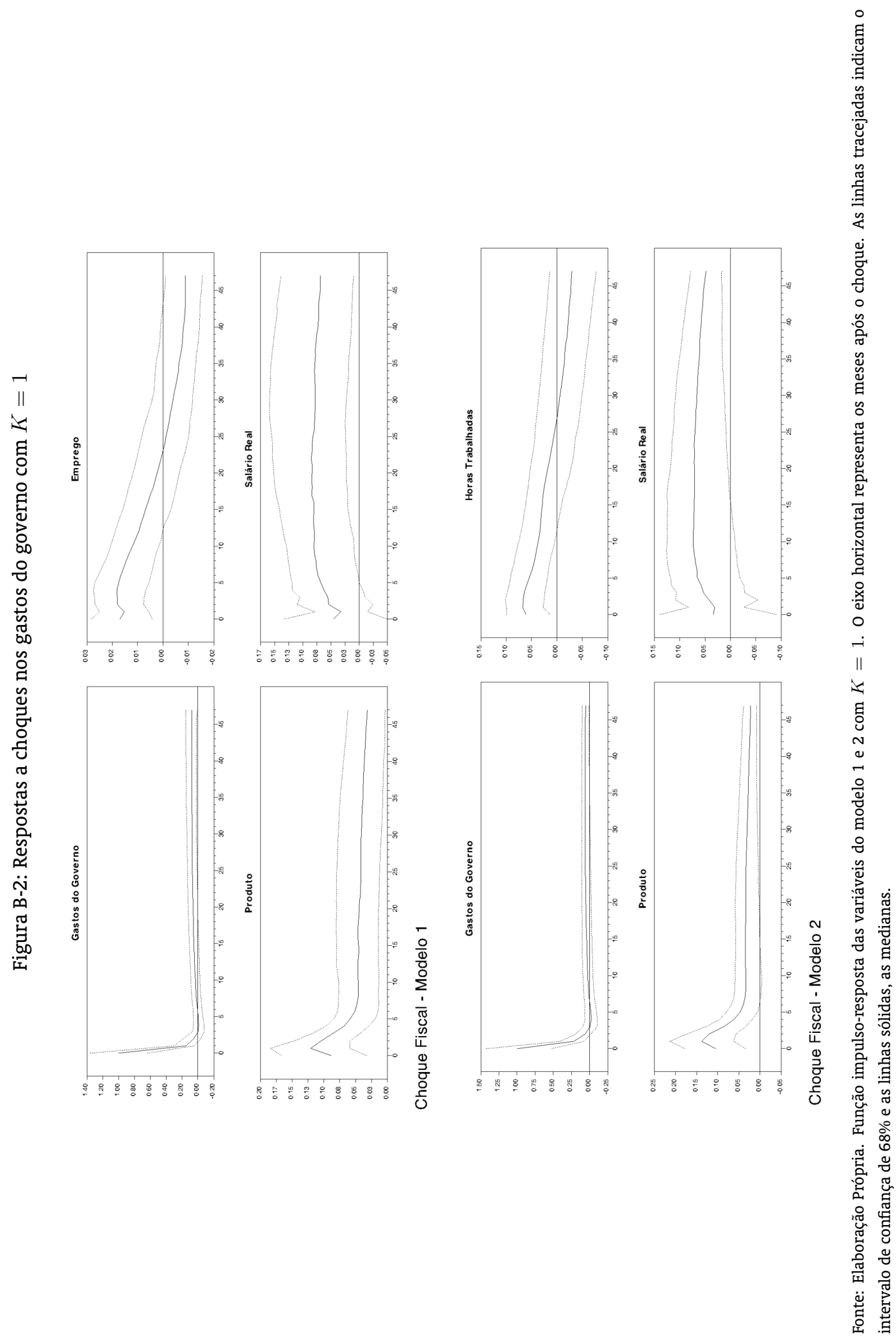

